\title{
Detection of 1,5-anhydroglucitol as a Biomarker for Diabetes Using an Organic Field-Effect Transistor-Based Biosensor
}

\author{
Hiroyuki Furusawa 1,2,*, Yusuke Ichimura 1,3, Kuniaki Nagamine ${ }^{1,3}$, Rei Shiwaku 1,3, \\ Hiroyuki Matsui ${ }^{1,3}$ and Shizuo Tokito ${ }^{1,3}$ \\ 1 Research Center for Organic Electronics (ROEL), Yamagata University, 4-3-16 Jonan, Yonezawa, \\ Yamagata 992-8510, Japan; tya85847@st.yamagata-u.ac.jp (Y.I.); nagamine@yz.yamagata-u.ac.jp (K.N.); \\ tdc57711@st.yamagata-u.ac.jp (R.S.); h-matsui@yz.yamagata-u.ac.jp (H.M.); \\ tokito@yz.yamagata-u.ac.jp (S.T.) \\ 2 Innovative Flex Course for Frontier Organic Material Systems (iFront), Graduate School of Science and \\ Engineering, Yamagata University, 4-3-16 Jonan, Yonezawa, Yamagata 992-8510, Japan \\ 3 Graduate School of Organic Materials Science, Yamagata University, 4-3-16 Jonan, Yonezawa, \\ Yamagata 992-8510, Japan \\ * Correspondence: hfurusaw@yz.yamagata-u.ac.jp; Tel.: +81-238-26-3841
}

Received: 19 July 2018; Accepted: 13 August 2018; Published: 15 August 2018

\begin{abstract}
Sensor devices that can be fabricated on a flexible plastic film produced at a low cost using inkjet-printing technology are suitable for point-of-care applications. An organic field-effect transistor (OFET)-based biosensor can function as a potentiometric electrochemical sensor. To investigate the usefulness of an OFET-based biosensor, we demonstrated the detection of 1,5-anhydroglucitol (1,5-AG) and glucose, which are monosaccharides used as biomarkers of diabetes. An OFET-based biosensor combined with a Prussian blue (PB) electrode, modified with glucose oxidase (GOx) or pyranose oxidase (POx), was utilized for the detection of the monosaccharides. When the GOx- or POx-PB electrode was immersed in glucose solution at the determined concentration, shifts in the low-voltage direction of transfer characteristic curves of the OFET were observed to be dependent on the glucose concentrations in the range of 0-10 mM. For 1,5-AG, the curve shifts were observed only with the POx-PB electrode. Detection of glucose and 1,5-AG was achieved in a substrate-specific manner of the enzymes on the printed OFET-biosensor. Although further improvements are required in the detection concentration range, the plastic-filmOFET-biosensors will enable the measurement of not only diabetes biomarkers but also various other biomarkers.
\end{abstract}

Keywords: organic field-effect transistor (OFET); diabetes biomarker; 1,5-anhydroglucitol; glucose; pyranose oxidase; glucose oxidase

\section{Introduction}

A biosensor, which allows for the detection of a target molecule specifically through biochemical reactions mediated by biofunctional materials like enzymes and antibodies, is in demand for environment monitoring, food analysis and healthcare [1-3]. Disease-related biomarkers, which include proteins and RNAs specific to patients with cancer [4,5], as well as enzymes and carbohydrates that show a characteristic trend in diabetic patients [6,7], have been identified by recent biomarker studies. Since the detection of disease-related biomarkers from human bodies could be an indicator for the early detection of the disease, a sensor that can detect biomarkers is useful for point-of-care applications in the healthcare sector [8]. The sensor devices can be designed to be handled easily at home, inexpensive and safe for humans and the environment. 
Among the various methods for detecting biomolecules, in general, optical sensing [9,10], mass sensing [11,12] and electrochemical sensing [13,14] are mainly used in sensors. Electrochemical measurements, including potentiometry and amperometry, allow for the construction of inexpensive, small and multi-channel biosensors by combining an enzyme electrode and an amplification circuit $[13,14]$. We focus on a point-of-care electrochemical biosensor. Previously, we had utilized an organic field-effect transistor (OFET), which uses an organic semiconducting material in an FET-type transistor [15], together with sensor devices. The OFET can function as a potentiometric device in electrochemical measurements [14]. In addition, an OFET-based biosensor offers advantages such as fabrication on a flexible plastic film and of multi-sensor arrays and a low cost of production, using inkjet-printing technology [16]. We have previously reported the OFET-based detection of biomolecules with a sensor for saccharides [17], lactic acid [18] and proteins [19] by using a chemical receptor of phenylboronic acid, an enzyme of lactate oxidase and an antibody.

Potentiometric detection by an OFET-based biosensor is based on the change of the surface potential of the sensing electrode [14]. When a gate electrode of the OFET is electrically connected to a DC voltage generator through a reference electrode, an electrolyte solution and a sensing surface on the sensor chip, a change in the sensing surface potential results in an apparent change in the gate electrode potential [15]. Oxidases produce hydrogen peroxide as a by-product in the oxidation reaction of the substrate. Since hydrogen peroxide can oxidize $\mathrm{Fe}^{2+}$ ions in Prussian blue (PB), that is, $\mathrm{Fe}_{4}\left[\mathrm{Fe}(\mathrm{CN})_{6}\right]_{3}$, into $\mathrm{Fe}^{3+}$ ions, an electrode containing $\mathrm{PB}$ can be used in combination with a sensor chip for monitoring an oxidase reaction via potentiometric measurements (Figure 1). In this study, we demonstrate the detection of 1,5-anhydroglucitol (1,5-AG) and glucose, which are monosaccharides used as diabetes biomarkers [20-22], using two OFET-based biosensors combined with a PB electrode modified with glucose oxidase (GOx) or pyranose oxidase (POx). The OFET device was fabricated by a printing process in our lab.

\section{Materials and Methods}

\subsection{Materials}

Polyvinylpyrrolidone (PVP, Mw $\approx 25,000$ ) and polystyrene (PS, Mw $\approx 280,000$ ) were purchased from Sigma-Aldrich Japan Co. (Tokyo, Japan). A silver nanoparticle ink in hydrocarbon-based solution (NPS-JL) was purchased from Harima Chemicals Group Inc. (Tokyo, Japan). Parylene (dix-SR) was obtained from KISCO Ltd. (Osaka, Japan). Fluoropolymer (Teflon AF1600) was purchased from DuPont-Mitsui Fluorochemicals Co., Ltd. (Tokyo, Japan). Pentafluorobenzenethiol (PFBT) was obtained from Tokyo Chemical Industry Co., Ltd. (Tokyo, Japan). 2,7-dihexyl-dithieno[2,3-d;2' '3'$\mathrm{d}^{\prime}$ ]benzo[1,2-b;4,5-b']dithiophene (DTBDT-C6), an organic semiconductor, was kindly provided by Tosoh Co. (Tokyo, Japan). A carbon graphite ink with PB was obtained from Gwent Electronic Materials Ltd. (Pontypool, UK). Chitosan (Mw > 100,000) was purchased from Junsei Chemical Co., Ltd (Tokyo, Japan). Pyranose oxidase (POx) from Coriolus sp. and Dulbecco's phosphate buffered saline (PBS) were purchased from Sigma-Aldrich Japan Co. (Tokyo, Japan). GOx from Aspergillus niger and glucose were purchased from Wako Pure Chemical Industries, Ltd. (Osaka, Japan). The compound 1,5-anhydroglucitol (1,5-AG) was purchased from Funakoshi Co., Ltd. (Tokyo, Japan). Other reagents were purchased from Sigma-Aldrich Japan Co. (Tokyo, Japan). Type 1 ultrapure water (Milli- $\mathrm{Q}^{\mathrm{TM}}$ water, Merck Ltd., Tokyo, Japan) was used in all the experiments.

\subsection{Fabrication of an OFET Device}

An OFET device, shown in Figure 1A, was fabricated as described in previous papers [23]. A silver electrode, acting as a gate electrode, was attached by printing with an inkjet printer (Dimatix DMP2381, Fujifilm Co., Tokyo, Japan) to a glass plate coated with cross-linked PVP for surface treatment, followed by heating at $120^{\circ} \mathrm{C}$ to sinter the silver nanoparticles. After coating the gate electrode with 70-nm-thick parylene by chemical vapor deposition, to form a dielectric layer, two silver electrodes, acting as the 
source and drain electrodes, were printed on the previously formed dielectric layer and then sintered in the same manner as the gate electrode. Using a dispenser system (Image Master 350 PC, Musashi Engineering, Inc., Tokyo, Japan), fluoropolymer solution (Teflon, $1 \mathrm{wt} \%$ ) was then printed in two parallel lines to form bank lines on both sides of a channel consisting of the source and drain electrodes. After the modification of the surface of the source and drain electrodes with the self-assembled monolayer of PFBT to reduce the contact resistance, a mixture solution of an organic semiconductor material, DTBDT-C6 $(0.9 \mathrm{wt} \%)$ and polystyrene $(0.3 \mathrm{wt} \%)$ in toluene, was printed onto the square area surrounded by the source and drain electrodes as well as the bank lines, followed by heating at $100{ }^{\circ} \mathrm{C}$ to remove the solvent. Finally, the fabricated device was covered with a Teflon layer by printing the fluoropolymer solution ( $1 \mathrm{wt} \%$ ) to improve the device stability. The fabricated OFET had a channel width of $780 \mu \mathrm{m}$ and a channel length of $13 \mu \mathrm{m}$ (Figure 1B).

(A)

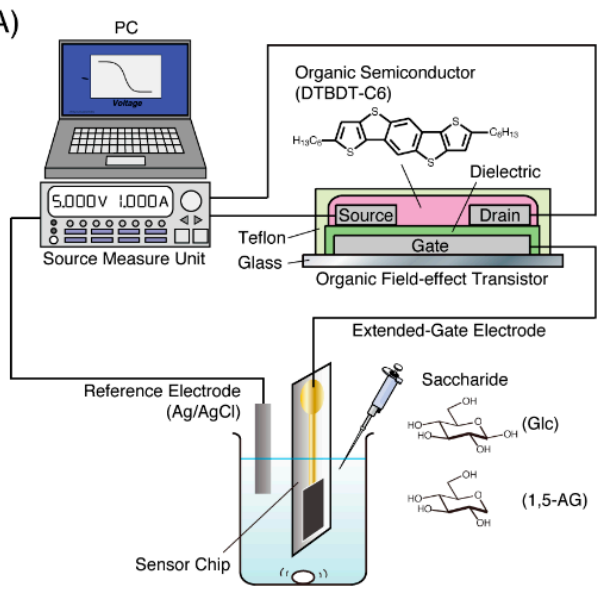

(B) Organic Semiconductor

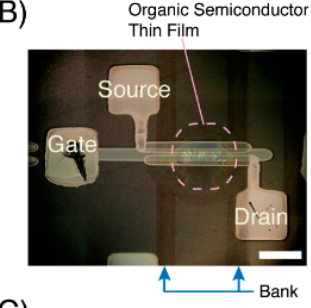

(C)

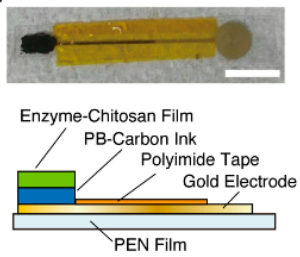

(D)
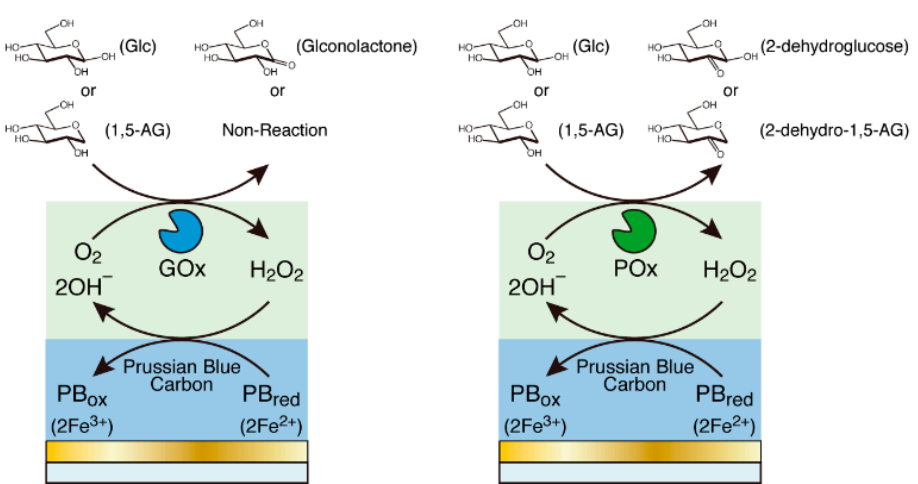

Figure 1. (A) An illustration of an organic field-effect transistor (OFET)-biosensor system using a sensor chip with an enzyme electrode for the potentiometric detection of saccharides. (B) A photograph of an OFET fabricated by the printing process obtained from the top of the substrate (white bar: $200 \mu \mathrm{m}$ ). The dashed circle indicates the OFET part. Squares indicated by Source, Drain and Gate are electrodes for contacting the external environment. Two parallel lines indicated by Bank on both sides of the dashed circle were printed to form a channel consisting of the source and drain electrodes. (C) A photograph and illustration of a sensor chip used in this study (white bar: $10 \mathrm{~mm}$ ). (D) Schematic illustrations of the reaction process of enzyme and Prussian blue in an enzyme electrode on the sensor chip.

\subsection{Preparation of an Enzyme Electrode for Biomarker Detection}

A polyethylene naphthalate (PEN) film of $125-\mu$ m thickness (Teonex, Teijin Ltd., Tokyo, Japan), on which a patterned gold thin film of 50-nm thickness was deposited to form an electrode, was used as a sensor chip in this study (Figure 1C). A sensing area of the gold electrode ( $3 \mathrm{~mm} \times 5 \mathrm{~mm}$ ) was 
coated with a carbon graphite ink with $\mathrm{PB}$ and then incubated at $30^{\circ} \mathrm{C}$ for $30 \mathrm{~min}$ for drying. Next, $11.4 \mu \mathrm{L}$ of a mixture consisting of $1.4 \mu \mathrm{L}$ of enzyme solution ( 1 unit $/ \mu \mathrm{L}$ of GOx or POx in $10 \times \mathrm{PBS})$ and $10 \mu \mathrm{L}$ of chitosan solution ( $0.1 \mathrm{wt} \%$ in aqueous solution at $\mathrm{pH}$ 5.4) was drop-casted onto the PB-carbon graphite coating and then, the sensor chip was incubated at $30^{\circ} \mathrm{C}$ for $3 \mathrm{~h}$, resulting in the formation of an enzyme-chitosan polyion complex film on the PB-carbon graphite coating. An enzyme-immobilized sensor chip was used for biomarker-detection experiments as an enzyme electrode.

\subsection{Measurement of 1,5-AG or Glucose Detection with an OFET-Based Biosensor}

A gold electrode of the enzyme-immobilized sensor chip and an $\mathrm{Ag} / \mathrm{AgCl}$ electrode (RE-1B, BAS Inc., Tokyo, Japan), as the reference electrode, were immersed in a 1.5-mL microtube filled with $0.5 \mathrm{~mL}$ of $1 \times$ PBS solution (Figure 1A). The gold electrode was connected to a gate electrode of the fabricated OFET device. The source and drain electrodes of the OFET device and the reference electrode were connected to a source measure unit (Keithley 2636B SYSTEM SourceMeter, Tektronix Inc., USA) for experiments using the OFET-based biosensor. First, we obtained the transfer characteristic curve of the OFET, which plots current values between the source and drain electrodes $\left(I_{\mathrm{DS}}\right)$ against gate voltage values ( $V_{\mathrm{g}}: 1$ to $-2 \mathrm{~V}, 0.05 \mathrm{~V}$ intervals) at a $-2 \mathrm{~V}$ potential between the source and drain electrodes $\left(V_{\mathrm{DS}}\right)$. Then, we observed curve shifts in response to the immersion of the electrode into the glucose or 1,5-AG solutions in the microtube.

\section{Results and Discussion}

\subsection{Characterization of the OFET Device}

To confirm the electrical characteristics of the fabricated OFET device, the transfer characteristic curve was measured by using a source measure unit in the $V_{\mathrm{g}}$ range of 1 to $-2 \mathrm{~V}$ at $V_{\mathrm{DS}}=-2 \mathrm{~V}$. The current values ( $\left.I_{\mathrm{DS}}\right)$ varied in six digits from the order of $10^{-12}$ to $10^{-6} \mathrm{~A}$ as OFF-ON currents of the transistor (Supplementary Materials Figure S1). This means that a large change in the current between the source and drain electrodes $\left(I_{\mathrm{DS}}\right)$ can be observed only with a slight change in the gate voltage value $\left(V_{\mathrm{g}}\right)$. Thus, the OFET-based biosensor with DTBDT-C6 as an organic semiconductor offers the advantage of higher sensitivity compared to our previous OFET sensor, which was equipped with another organic semiconductor poly(2,5-bis(3-hexadecylthiophen-2-yl)thieno[3,2b] thiophene)(PBTTT- $\mathrm{C}_{16}$ ) with ON-OFF currents of a transistor in the order of $10^{-9}$ to $10^{-6} \mathrm{~A}[15,17]$.

\subsection{Observation of the Transfer Characteristic Curves of the OFET Equipped with a GOx-PB Electrode}

GOx catalyzes the oxidation of D-glucose to D-glucono-1,5-lactone, which indicates the transformation of a hydroxyl group to a ketone group at the 1-position of D-glucose (Figure 1D). Because 1,5-AG has a dehydroxylated structure at the 1-position of D-glucose, GOx cannot react with 1,5-AG. We observed the enzyme reactivity of glucose and 1,5-AG to confirm that the enzyme electrodes function as a sensor.

A sensor chip with a GOx-PB electrode was connected to the gate electrode of the OFET and immersed in a microtube with PBS solution. Then, the OFET device was set up with connections to a source measure unit in order to obtain the transfer characteristic curve. When the GOx-PB electrode was immersed in glucose solution of the determined concentrations, shifts in the low-voltage direction of the transfer characteristic curves were observed to be dependent on the glucose concentrations in the range of 0 to $10 \mathrm{mM}$, as shown in Figure 2A. The observed shifts were as expected. Hydrogen peroxide produced from oxidation by GOx increased the number of $\mathrm{Fe}^{3+}$ ions in $\mathrm{PB}$ on the sensor surface, resulting in an increase in the potential of the PB electrode to the reference electrode. Thus, in order to obtain the same $I_{\mathrm{DS}}$ value, it was necessary to apply a lower gate voltage. On the other hand, no curve shifts were observed when 1,5-AG solution was used in the same manner (Figure 2B). These results suggest that the OFET response to glucose solution could be caused by an oxidation reaction of GOx in a substrate-specific manner of the enzyme. 
(A)

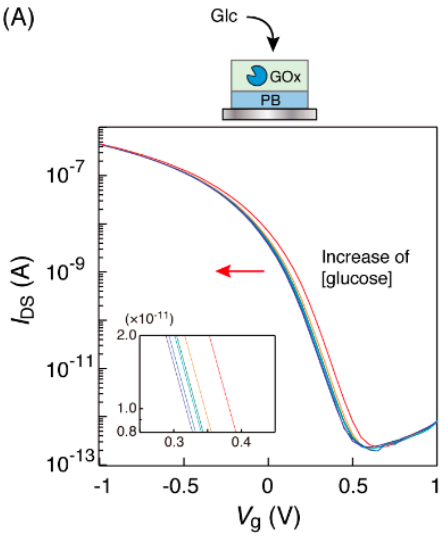

(B)

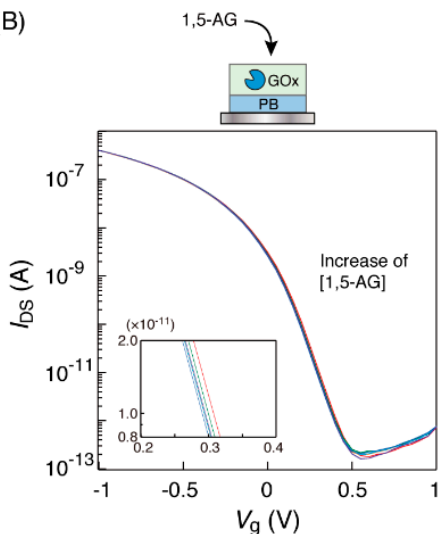

Figure 2. Transfer characteristic curves of the organic field-effect transistor (OFET) biosensor with a GOx-PB electrode obtained in response to the electrode's immersion into (A) glucose and (B) 1,5 AG solutions at different concentrations $(0,0.1,0.5,1,3$ and $10 \mathrm{mM})$, buffered by $1 \times$ PBS at $25{ }^{\circ} \mathrm{C}$. Each concentration curve has been indicated in red $(0 \mathrm{mM})$, orange $(0.1 \mathrm{mM})$, green $(0.5 \mathrm{mM})$, light blue $(1 \mathrm{mM})$, blue $(3 \mathrm{mM})$ and purple $(10 \mathrm{mM})$, respectively. The insets show the enlarged views.

\subsection{Observation of the Transfer Characteristic Curves of The OFET Equipped with A POx-PB Electrode}

POx is an enzyme that catalyzes the oxidation of D-glucose to 2-dehydro-D-glucose, indicating the transformation of the hydroxyl group to the ketone group at the 2-position (Figure 1D). Thus, POx can oxidize both 1,5-AG and D-glucose. We confirmed that a POx-PB electrode can detect glucose and 1,5-AG, as shown by a shift of the transfer characteristic curve in the same manner as that for a GOx-PB electrode.

The immersion of the POx-PB electrode into the glucose solution in the microtube caused a low-voltage direction shift of the transfer characteristic curves (Figure 3A). Similar curve shifts were also observed in case of the 1,5-AG solution for the POx-PB electrode (Figure 3B). The amounts of these curve shifts were approximately $60 \mathrm{mV}$, in the concentration range of 0 to $10 \mathrm{mM}$ of the saccharides; these curve shifts were in the same direction and were of similar values as those for the detection of glucose with the GOx-PB electrode. These results indicated that POx on the electrode could oxidize 1,5-AG and D-glucose, with the production of hydrogen peroxide, which converted the $\mathrm{Fe}^{2+}$ ions in the $\mathrm{PB}$ on the electrode into $\mathrm{Fe}^{3+}$ ions.
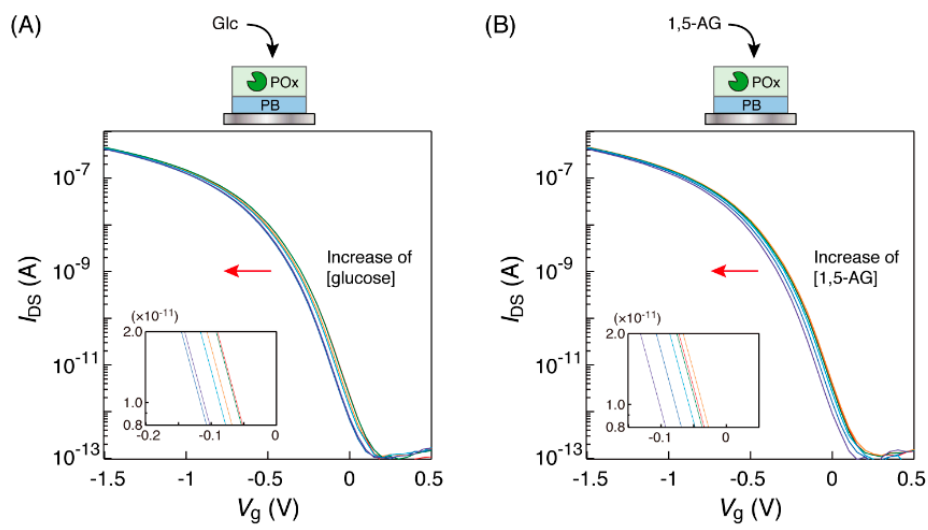

Figure 3. Transfer characteristic curves of the organic field-effect transistor (OFET) biosensor with a POx-PB electrode obtained in response to the electrode's immersion into (A) glucose and (B) 1,5-AG solutions at different concentrations $(0,0.1,0.5,1,3$ and $10 \mathrm{mM})$, buffered by $1 \times \mathrm{PBS}$ at $25{ }^{\circ} \mathrm{C}$. Each concentration curve has been indicated in red $(0 \mathrm{mM})$, orange $(0.1 \mathrm{mM})$, green $(0.5 \mathrm{mM})$, light blue $(1 \mathrm{mM})$, blue $(3 \mathrm{mM})$ and purple $(10 \mathrm{mM})$, respectively. The insets show the enlarged views. 
In addition, other monosaccharides, such as mannose and galactose, were measured to confirm the substrate-specific detection of the POx-PB electrode. Mannose is a stereoisomer (a 2-position epimer) of glucose and is not a substrate for POx. In fact, the shifts of the transfer characteristic curves were not observed as shown in Figure 4A, when the POx-PB electrode was immersed in mannose solution. Galactose is a 4-position epimer of glucose and POx does not catalyze its oxidation. We observed no shifts of the transfer characteristic curves to the low-voltage direction (Figure 4B). These results suggest that the curve shifts should be based on the reaction of the monosaccharides in the solution with the enzyme on the sensor chip. Thus, by using the OFET-biosensor, we could detect a monosaccharide in the solution as an electropotential change of the PB electrode.
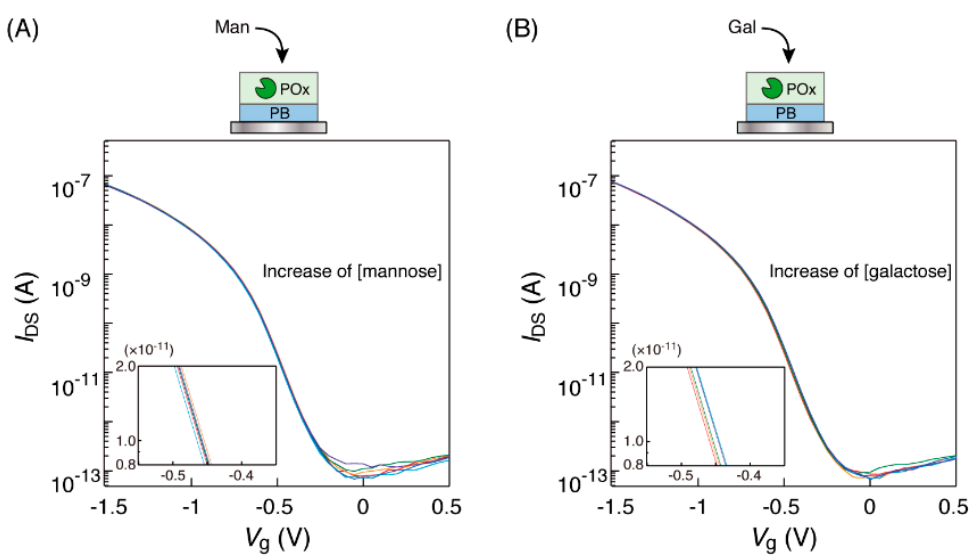

Figure 4. Transfer characteristic curves of the organic field-effect transistor (OFET) biosensor with a POx-PB electrode obtained in response to the electrode's immersion into (A) mannose and (B) galactose solutions at different concentrations $(0,0.1,0.5,1,3$ and $10 \mathrm{mM})$, buffered by $1 \times \mathrm{PBS}$ at $25{ }^{\circ} \mathrm{C}$. Each concentration curve has been indicated in red $(0 \mathrm{mM})$, orange $(0.1 \mathrm{mM})$, green $(0.5 \mathrm{mM})$, light blue $(1 \mathrm{mM})$, blue $(3 \mathrm{mM})$ and purple $(10 \mathrm{mM})$, respectively. The insets show the enlarged views.

\subsection{Relationship between Saccharide Concentration and Curve Shift}

We investigated the relationship between the saccharide concentrations in the solution and the curve shift of the OFET-biosensor. Figure 5 shows the plots of the $V_{\mathrm{g}}$ value shifts $\left(\Delta V_{\mathrm{g}}\right)$ as a function of the concentration of each monosaccharide in the detection experiments using the OFET biosensor. For matched combinations of an enzyme and a substrate, saturation curves with a maximum $V_{\mathrm{g}}$ value of approximately $60 \mathrm{mV}$ were shown (Figure $5 \mathrm{~A}(\mathrm{a}), \mathrm{B}(\mathrm{a}, \mathrm{b})$ ). On the other hand, the $V_{\mathrm{g}}$ value shifts for curves of the combination where the enzymes and saccharides were not matched were within $\pm 20 \mathrm{mV}$.

Detection of glucose and 1,5-AG was achieved by the printable OFET biosensor. However, in consideration of the signal/noise ratio, the detected concentration range was approximately $1 \mathrm{mM}$ or more. Our strategy to evaluate 1,5-AG in the blood was to use the difference between the two sensors: "glucose" measurements by the GOx-PB electrode and "glucose + 1,5-AG" measurements by the POx-PB electrode. The concentration of 1,5-AG in the blood of a normal person is approximately $20 \mu \mathrm{g} / \mathrm{mL}(120 \mu \mathrm{M})$ [21]. In order to perform the measurement with an actual sample at home, it is necessary to improve the signal/noise ratio by one digit or more. We should consider combinations with noise reduction circuits [23] and/or efforts to increase the number of scans to obtain $\Delta V_{\mathrm{g}}$ values, in order to reduce the influence of random fluctuation. 
(A)

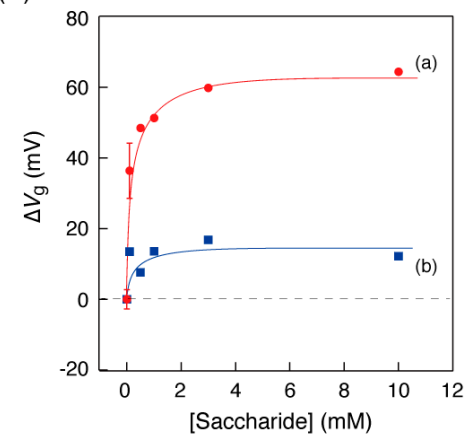

(B)

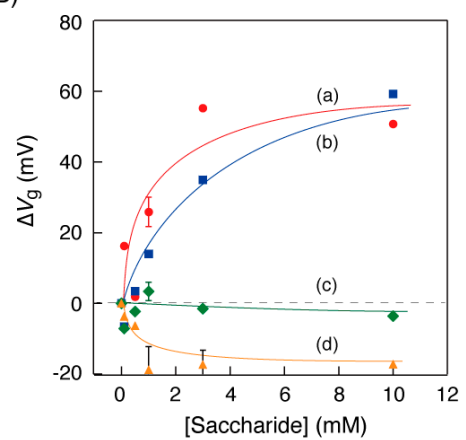

Figure 5. Shifts of the gate voltage $\left(\Delta V_{\mathrm{g}}\right.$ at $\left.I_{\mathrm{DS}}=10^{-11} \mathrm{~A}\right)$ in case of $(\mathrm{A})$ the GOx-PB electrode, depending on the concentration of (a: circle) glucose and (b: square) 1,5-AG and in case of (B) the POx-PB electrode, depending on the concentration of (a: circle) glucose, (b: square) 1,5-AG, (c: diamond) mannose and (d: triangle) galactose in $1 \times \mathrm{PBS}$ at $25^{\circ} \mathrm{C}$. The error bars indicate the standard deviation of three or four measurements.

\section{Conclusions}

We demonstrated the detection of glucose and 1,5-AG, two biomarkers for diabetes, using an OFET device equipped with a GOx- or POx-PB electrode. The monosaccharides could be detected by potentiometric measurements in the concentration range of $1 \mathrm{mM}$ or more. Further improvements are required for measurements of such monosaccharides/biomarkers in actual samples. We believe that plastic-film biosensors based on an OFET, which can be obtained inexpensively through the printing process, will enable the measurements of the biomarkers of not only diabetes but also various other diseases at home, for healthcare purposes.

Supplementary Materials: The following are available online at http://www.mdpi.com/2227-7080/6/3/77/s1, Figure S1: Fundamental transfer characteristic curves of the OFET biosensor in the $V_{\mathrm{g}}$ range of 1 to $-2 \mathrm{~V}$.

Author Contributions: H.F. supervised the entire project and wrote the manuscript. Y.I. performed the measurements and analysis of the OFET experiments. R.S. and H.M. fabricated the OFET device and advised on the device handling. K.N. and S.T. participated in comprehensive discussion and provided a helpful advice and suggestions.

Funding: This research was funded by Nakayama Foundation for Human Science.

Acknowledgments: The authors acknowledge Shin-ichi Wakida of the National Institute of Advanced Industrial Science and Technology (AIST) and Osamu Niwa of the Saitama Institute of Technology for their valuable discussion. The authors also acknowledge Tosoh Corporation for providing the organic semiconductor used in this study. The authors thank Daisuke Kumaki, Yasunori Takeda, Kazuhiko Tsuchiya and Ayako Nomura of Yamagata University for their technical support and advice.

Conflicts of Interest: The authors declare no conflict of interest.

\section{References}

1. Karlsson, R. SPR for molecular interaction analysis: A review of emerging application areas. J. Mol. Recognit. 2004, 17, 151-161. [CrossRef] [PubMed]

2. Mohanty, S.P.; Kougianos, E. Biosensors: A tutorial review. IEEE Potentials 2006, 25, 35-40. [CrossRef]

3. Yakovleva, M.; Bhand, S.; Danielsson, B. The enzyme thermistor-A realistic biosensor concept. A critical review. Anal. Chim. Acta 2013, 766, 1-12. [CrossRef] [PubMed]

4. Srinivas, P.R.; Verma, M.; Zhao, Y.; Srivastava, S. Proteomics for Cancer Biomarker Discovery. Clin. Chem. 2002, 48, 1160-1169. [PubMed]

5. Hayes, J.; Peruzzi, P.P.; Lawler, S. MicroRNAs in cancer: Biomarkers, functions and therapy. Trends Mol. Med. 2014, 20, 460-469.

6. Matheson, A.; Willcox, M.D.P.; Flanagan, J.; Walsh, B.J. Urinary biomarkers involved in type 2 diabetes: A review. Diabetes Metab. Res. Rev. 2010, 26, 150-171. [CrossRef] [PubMed] 
7. Suhre, K.; Meisinger, C.; Döring, A.; Altmaier, E.; Belcredi, P.; Gieger, C.; Chang, D.; Milburn, M.V.; Gall, W.E.; Weinberger, K.M.; et al. Metabolic Footprint of Diabetes: A Multiplatform Metabolomics Study in an Epidemiological Setting. PLoS ONE 2010, 5, e13953. [CrossRef] [PubMed]

8. Jani, I.V.; Peter, T.F. How Point-of-Care Testing Could Drive Innovation in Global Health. N. Engl. J. Med. 2013, 368, 2319-2324. [CrossRef] [PubMed]

9. Homola, J.; Yee, S.S.; Gauglitz, G. Surface plasmon resonance sensors: Review. Sens. Actuators B Chem. 1999, 54, 3-15. [CrossRef]

10. Addas, A.; Linman, M.J.; Cheng, Q. New trends in instrumental design for surface plasmon resonance-based biosensors. Biosens. Bioelectron. 2011, 26, 1815-1824.

11. Becker, B.; Cooper, M.A. A survey of the 2006-2009 quartz crystal microbalance biosensor literature. J. Mol. Recognit. 2011, 24, 754-787. [CrossRef] [PubMed]

12. Alassi, A.; Benammar, M.; Brett, D. Quartz Crystal Microbalance Electronic Interfacing Systems: A Review. Sensors 2017, 17, 2799. [CrossRef] [PubMed]

13. Wang, J. Electrochemical biosensors: Towards point-of-care cancer diagnostics. Biosens. Bioelectron. 2006, 21, 1887-1892. [CrossRef] [PubMed]

14. Grieshaber, D.; MacKenzie, R.; Vörös, J.; Reimhult, E. Electrochemical Biosensors - Sensor Principles and Architectures. Sensors 2008, 8, 1400-1458. [CrossRef] [PubMed]

15. Furusawa, H.; Ichimura, Y.; Harada, S.; Uematsu, M.; Xue, S.; Nagamine, K.; Tokito, S. Electric Charge Detection of Sparse Organic Acid Molecules Using an Organic Field-Effect Transistor (OFET)-Based Sensor. Bull. Chem. Soc. Jpn. 2018, 91, 1020-1025. [CrossRef]

16. Someya, T.; Sekitani, T.; Iba, S.; Kato, Y.; Kawaguchi, H.; Sakurai, T. A large-area, flexible pressure sensor matrix with organic field-effect transistors for artificial skin applications. Proc. Natl. Acad. Sci. USA 2004, 101, 9966-9970. [CrossRef] [PubMed]

17. Minami, T.; Minamiki, T.; Hashima, Y.; Yokoyama, D.; Sekine, T.; Fukuda, K.; Kumaki, D.; Tokito, S. An extended-gate type organic field effect transistor functionalized by phenylboronic acid for saccharide detection in water. Chem. Commun. 2014, 50, 15613-15615. [CrossRef] [PubMed]

18. Minami, T.; Sato, T.; Minamiki, T.; Fukuda, K.; Kumaki, D.; Tokito, S. A novel OFET-based biosensor for the selective and sensitive detection of lactate levels. Biosens. Bioelectron. 2015, 74, 45-48. [CrossRef] [PubMed]

19. Minamiki, T.; Minami, T.; Sasaki, Y.; Wakida, S.; Kurita, R.; Niwa, O.; Tokito, S. Label-free detection of human glycoprotein (CgA) using an extended-gated organic transistor-based immunosensor. Sensors 2016, 16, 2033. [CrossRef] [PubMed]

20. Yamanouchi, T.; Minoda, S.; Yabuuchi, M.; Akanuma, Y.; Akanuma, H.; Miyashita, H.; Akaoka, I. Plasma 1,5-anhydro-D-glucitol as new clinical marker of glycemic control in NIDDM patients. Diabetes 1989, 38, 723-729. [CrossRef] [PubMed]

21. Nowatzke, W.; Sarno, M.J.; Birch, N.C.; Stickle, D.F.; Eden, T.; Cole, T.G. Evaluation of an assay for serum 1,5-anhydroglucitol (GlycoMark ${ }^{\mathrm{TM}}$ ) and determination of reference intervals on the Hitachi 917 analyzer. Clin. Chem. Acta 2004, 350, 201-209. [CrossRef] [PubMed]

22. Fukumura, Y.; Tajima, S.; Oshitani, S.; Ushijima, Y.; Kobayashi, I.; Hara, F.; Yamamoto, S.; Yabuuchi, M. Fully enzymatic method for determining 1,5-anhydro-D-glucitol in serum. Clin. Chem. 1994, 40, 2013-2016. [PubMed]

23. Shiwaku, R.; Matsui, H.; Nagamine, K.; Uematsu, M.; Mano, T.; Maruyama, Y.; Nomura, A.; Tsuchiya, K.; Hayasaka, K.; Takeda, Y.; et al. A Printed Organic Circuit System for Wearable Amperometric Electrochemical Sensors. Sci. Rep. 2018, 8, 6368. [CrossRef] [PubMed]

(C) 2018 by the authors. Licensee MDPI, Basel, Switzerland. This article is an open access article distributed under the terms and conditions of the Creative Commons Attribution (CC BY) license (http:// creativecommons.org/licenses/by/4.0/). 